Z Rheumatol 2018· 77:429-441 https://doi.org/10.1007/s00393-018-0476-8 Online publiziert: 30. Mai 2018

(c) Deutsche Gesellschaft für Rheumatologie e.V. Published by Springer Medizin Verlag $\mathrm{GmbH}$. All rights reserved 20182018

CrossMark
Beteiligte medizinisch-wissenschaftliche Fachgesellschaften und weitere Organisationen

Deutsche Gesellschaft für Rheumatologie (DGRh)

Österreichische Gesellschaft für Rheumatologie und Rehabilitation (ÖGR)

Schweizerische Gesellschaft für Rheumatologie (SGR)

Deutsche Gesellschaft für Geriatrie (DGG)

Deutsche Rheuma-Liga

Deutsche Gesellschaft für Innere Medizin (DGIM)

Deutsche Gesellschaft für Orthopädie und

Orthopädische Chirurgie (DGOOC)

Deutsche Gesellschaft für Physikalische Medizin und Rehabilitation (DGPMR)

F. Buttgereit ${ }^{1}$ für DGRh $\cdot$ T. Brabant ${ }^{2}$ für DGG $\cdot$ H. Dinges ${ }^{3}$ für DGOOC $\cdot$ I. Hiemer ${ }^{4}$ für Rheuma-Liga · M. Kaplani ${ }^{5}$ für DGIM · U. Kiltz ${ }^{6}$ D. Kyburz für SGR · A. Reißhauer ${ }^{8}$ für DGPMR · M. Schneider ${ }^{9}$ für DGRh · C. Weseloh ${ }^{10} \cdot$ C. Dejaco ${ }^{11,12}$ für ÖGR 'Medizinische Klinik mit Schwerpunkt Rheumatologie und Klinische Immunologie, Charité Universitätsmedizin Berlin, Berlin, Deutschland; ${ }^{2}$ Zentrum für Geriatrie und Frührehabilitation, Krankenhaus Sankt-Joseph-Stift, Bremen, Deutschland; ${ }^{3}$ Klinik für Orthopädie - Gelenkersatz - Rheuma - Unfallchirurgie, Westpfalz-Klinikum GmbH Kaiserslautern/Kusel, Kusel, Deutschland; ${ }^{4}$ Hamburg, Deutschland; ${ }^{5}$ Gemeinschaftspraxis, Berlin, Deutschland; ${ }^{6}$ Rheumazentrum Ruhrgebiet, Herne, Deutschland; ${ }^{7}$ Klinik für Rheumatologie, Universitätsspital Basel, Basel, Schweiz; ${ }^{8}$ Arbeitsbereich Physikalische Medizin, Charité Universitätsmedizin Berlin, Berlin, Deutschland; ${ }^{9}$ Poliklinik und Funktionsbereich für Rheumatologie, Universitätsklinikum Düsseldorf, Heinrich-Heine-Universität, Düsseldorf, Deutschland; ${ }^{10}$ Deutsche Gesellschaft für Rheumatologie e. V., Berlin, Deutschland; "Dienst für Rheumatologie, Südtiroler Sanitätsbetrieb, Bruneck, Italien; ${ }^{12}$ Medizinische Universität Graz, Graz, Österreich

\title{
S3-Leitlinie zur Behandlung der Polymyalgia rheumatica
}

\section{Evidenzbasierte Leitlinie der Deutschen Gesellschaft für Rheumatologie (DGRh), der Österreichischen Gesellschaft für Rheumatologie und Rehabilitation (ÖGR) und der Schweizerischen Gesellschaft für Rheumatologie (SGR) und der beteiligten medizinisch-wissenschaftlichen Fachgesellschaften und weiterer Organisationen}

Die Polymyalgia rheumatica (PMR) ist die zweithäufigste entzündlich rheumatische Autoimmunerkrankung bei Personen im höheren Lebensalter nach der rheumatoiden Arthritis [1]. Da es für die PMR keinen spezifischen Nachweis gibt, ist die Erkrankung nicht immer leicht zu diagnostizieren, und so lässt sich die Diagnose oft erst nach Ausschluss klinisch ähnelnder Differenzialdiagnosen stellen [2]. Die PMR tritt fast ausschließlich bei Menschen oberhalb des 50. Lebensjahres auf, wobei Frauen etwa 3-mal häufiger betroffen sind als Männer. Wichtig ist, dass die PMR überzufällig häufig gemeinsam mit einer Riesenzellarteriitis auftreten kann, was dann eine Therapieeskalation zur Folge hat [1].

\begin{tabular}{|l}
\hline Infobox \\
\hline AWMF-Leitlinien Register Nummer: 060/006 \\
Entwicklungsstufe:S3 \\
Convenor/Leitliniensekretariat: Prof. Dr. Frank \\
Buttgereit \\
Methodische Beratung: Dr. Uta Kiltz, \\
C. Weseloh \\
Besonderer Hinweis: Die Medizin unterliegt \\
einem fortwährenden Entwicklungsprozess, \\
sodass alle Angaben, insbesondere zu \\
therapeutischen Verfahren, immer nur dem \\
Wissensstand zur Zeit der Drucklegung der \\
Leitlinie entsprechen können. Hinsichtlich der \\
angegebenen Empfehlungen zur Therapie \\
wurde die größtmögliche Sorgfalt beachtet. \\
Der Benutzer selbst bleibt verantwortlich \\
für jede diagnostische und therapeutische \\
Applikation, Medikation und Dosierung.
\end{tabular}


Die Ätiopathogenese der PMR ist noch nicht vollständig geklärt. Es wird jedoch vermutet, dass genetische Faktoren, Infektionen, Alterungsprozesse des Immun- und Gefäßsystems und Störungen endokriner Achsen zur Entstehung und Ausprägung der Erkrankung beitragen. Möglichweise handelt es sich bei der PMR um eine frühe, subklinische Vaskulitis mit prominenter systemischer, artikulärer und periartikulärer Entzündungssymptomatik [1].

Klinisch stehen bei der PMR bilaterale Schulterschmerzen im Vordergrund, über die bis zu $95 \%$ der Patienten berichten [3]. Ebenfalls sehr häufig sind Nackenschmerzen und/oder Schmerzen im Beckengürtelbereich, die meist akut oder subakut auftreten. Charakteristisch ist auch eine ausgeprägte Morgensteifigkeit. Des Weiteren treten Gelenkentzündungen und Tenosynovitiden sowohl an proximalen (Schultern, Hüften) als auch distalen Gelenken (Hände, Knie) auf, ebenso Allgemeinsymptome wie Fieber, Appetitlosigkeit, Schwäche und/oder Gewichtsverlust $[1,4]$. Eine PMR wird diagnostiziert, wenn typische Symptome bzw. klinische Befunde in Kombination mit entsprechenden Laborergebnissen (in der Regel ist die Blutsenkungsgeschwindigkeit beschleunigt und/oder das C-reaktive Protein erhöht) vorliegen [3]. Befunde in der bildgebenden Diagnostik wie eine Bursitis subdeltoidea, Bizepssehnentenosynovitis und/oder eine Synovitis der Glenohumeralgelenke erhöhen die diagnostische Sicherheit [3]. Als relevante Differenzialdiagnosen sollten u. a. die rheumatoide Arthritis des höheren Lebensalters, Riesenzellarteriitis, Chondrokalzinose, Infektionen und Malignome in Betracht gezogen werden $[1,2]$.

\section{Begründung für die Erstellung der Leitlinien}

Diese Leitlinie wurde erarbeitet, weil trotz der relativ großen Anzahl an PMR erkrankter Patientinnen und Patienten ein sehr heterogenes Vorgehen im deutschsprachigen Sprachraum (aber auch europa- und weltweit) bei der Behandlung existiert.

\section{Ziel und Adressaten der Leitlinie}

Diese Leitlinie soll unter Berücksichtigung der derzeitig vorliegenden Evidenz als Unterstützung und Entscheidungshilfe bei der Behandlung von PMR-Patienten in der klinischen Praxis dienen. Die individuellen Behandlungsentscheidungen obliegen dem betreuenden Arzt. Bei dieser Leitlinie steht die Frage im Mittelpunkt, wie bei Patientinnen und Patienten mit dieser Erkrankung pharmakologische und nichtpharmakologische Maßnahmen angewandt werden sollen, um ein bestmögliches Nutzen-Risiko-Verhältnis bei der Behandlung zu erzielen. Im Sinne der besseren Lesbarkeit sprechen wir nachfolgend nicht immer wieder von "Patientinnen und Patienten“, sondern sehen ,den Patienten“als die Zusammenfassung von Männern und Frauen. Die Patientenzielgruppe umfasst alle erwachsenen Patienten mit einer klinisch gesicherten PMR. Die Leitlinie richtet sich an alle Ärzte/Ärztinnen, die Patienten mit PMR betreuen, primär an Rheumatologen sowie zur Information auch an andere Ärzte und Angehörige nichtärztlicher Berufsgruppen, die sich direkt oder indirekt an der Betreuung von Patienten mit PMR beteiligen. Die Leitlinie kann darüber hinaus zur Orientierung für an einer PMR erkrankte Patienten und deren Angehörige dienen.

\section{Methoden zur Erstellung der S3- Leitlinien}

Vor dem Hintergrund der 2015 publizierten Quellleitlinie, den 2015 EULARACR-Empfehlungen zum Management der Polymyalgia rheumatica (PMR) [5, 6], wurde diese hier vorliegende S3-Leitlinie im Auftrag der Deutschen Gesellschaft für Rheumatologie (DGRH) und in Kooperation mit der Österreichischen Gesellschaft für Rheumatologie und Rehabilitation (ÖGR) und der Schweizerischen Gesellschaft für Rheumatologie (SGR) erarbeitet. Aus diesem Grund setzte sich die Leitlinienkommission wie folgt zusammen: je 1 Vertreter der vorgenannten Gesellschaften, je 1 Delegierte(r) der Deutschen Gesellschaft für Geriatrie (DGG), der Deutschen Rheuma-Liga, der Deutschen Gesellschaft für
Innere Medizin (DGIM), der Deutschen Gesellschaft für Orthopädie und Orthopädische Chirurgie (DGOOC) sowie der Deutschen Gesellschaft für Physikalische Medizin und Rehabilitation (DGPMR). Als Moderatorin fungierte Frau Uta Kiltz, beratend standen der Kommission Matthias Schneider (Präsident der DGRh 2013-14 und Leitlinienbeauftragter der DGRh) und Christiane Weseloh (wissenschaftliche Mitarbeiterin der DGRh) zur Seite. Ein(e) Vertreter/in der Fachassistenz konnte ebenso wenig wie ein(e) Delegierte(r) der Deutschen Gesellschaft für Allgemeinmedizin für die Mitarbeit gewonnen werden. Die Leitlinienkommission konstituierte sich anlässlich des ersten Treffens im April 2016, legte sich auf das grundsätzliche Vorgehen fest und formulierte die Schlüsselfragen. Das zweite Treffen fand im Februar 2017 statt, um im Ergebnis der Literaturarbeit die Empfehlungen zu diskutieren und zu konsentieren.

Bei der Erarbeitung dieser S3-Leitlinie wurden dieselben Methoden angewandt wie zur Entwicklung der Quellleitlinie, den 2015 EULAR-ACR-Empfehlungen zum Management der Polymyalgia rheumatica (PMR) [5, 6]. Die Entwicklung der Empfehlungen basierte dabei auf der Grading of Recommendations Assessment, Development and Evaluation(GRADE)-Methode [7]. Die Schlüsselfragen („key questions“), die die Grundlage für die systematische Literatursuche (SLR) bildeten, wurden im sog. PICO-Format (=Population, Intervention, Comparator, Outcome) formuliert. Für die 2015 EULAR-ACREmpfehlungen wurden diese durch eine internationale Expertenkommission, bestehend aus männlichen und weiblichen Rheumatologen, Internisten, Allgemeinmedizinern, Vertretern der rheumatologischen Gesundheitsberufe (",health care professionals") und Patienten, entwickelt. Für die aktuelle S3-Leitlinie wurden die Schlüsselfragen von der Leitlinienkommission im Ergebnis einer ausführlichen Diskussion komplett übernommen.

Insgesamt wurden 12 PICO-Fragen zu Interventionen und 10 PICO-Fragen zu Prognosefaktoren entwickelt. Diese sind in - Tab. 1 dargestellt. Die Liste der „Outcomes", also der Ergebnispa- 
Z Rheumatol 2018· 77:429-441 https://doi.org/10.1007/s00393-018-0476-8

(c) Deutsche Gesellschaft für Rheumatologie e.V. Published by Springer Medizin Verlag GmbH. All rights reserved 20182018

F. Buttgereit für DGRh · T. Brabant für DGG · H. Dinges für DGOOC • I. Hiemer für Rheuma-Liga · M. Kaplani für DGIM • U. Kiltz • D. Kyburz für SGR · A. Reißhauer für DGPMR · M. Schneider für DGRh · C. Weseloh · C. Dejaco für ÖGR

\section{S3-Leitlinie zur Behandlung der Polymyalgia rheumatica. Evidenzbasierte Leitlinie der Deutschen} Gesellschaft für Rheumatologie (DGRh), der Österreichischen Gesellschaft für Rheumatologie und Rehabilitation (ÖGR) und der Schweizerischen Gesellschaft für Rheumatologie (SGR) und der beteiligten medizinisch-wissenschaftlichen Fachgesellschaften und weiterer Organisationen

\section{Zusammenfassung}

Die Polymyalgia rheumatica (PMR) tritt fast ausschließlich bei Menschen über 50 Jahren auf und ist nach der rheumatoiden Arthritis die zweithäufigste entzündlich rheumatische Erkrankung im höheren Lebensalter. Da spezifische Tests für die Erkrankung fehlen, wird die Diagnose oft erst nach Ausschluss klinisch ähnlicher Differenzialdiagnosen gestellt. Diese Leitlinie zur Behandlung der PMR setzt voraus, dass die Diagnose einer PMR bereits gestellt worden ist. Unmittelbar nach Diagnosestellung soll eine Therapie mit
Glukokortikoiden eingeleitet werden und eine angemessene Patientenschulung in Bezug auf die Auswirkungen der PMR und deren Behandlung erfolgen. Methotrexat kann bei Patienten mit hohem Risiko für Rezidive und/oder Glukokortikoidnebenwirkungen eingesetzt werden. Diese Leitlinie wurde erarbeitet, weil trotz der relativ großen Anzahl an Patienten mit PMR das Vorgehen zur Behandlung dieser Erkrankung im deutschsprachigen Sprachraum (aber auch europa- und weltweit) sehr heterogen ist. Als
Quellleitlinie dienten die 2015 publizierten EULAR-ACR-Empfehlungen zum Management der Polymyalgia rheumatica, die durch die Leitlinienkommission aktualisiert und auf den deutschen Sprachraum angepasst wurden.

Schlüsselwörter

Polymyalgia rheumatica . Patientenschulung . Glukokortikoide · Methotrexat · EULAR-ACREmpfehlungen

\section{S3 guidelines on treatment of polymyalgia rheumatica. Evidence-based guidelines of the German Society of Rheumatology (DGRh), the Austrian Society of Rheumatology and Rehabilitation (ÖGR) and the Swiss Society of Rheumatology (SGT) and participating medical scientific specialist societies and other organizations}

\begin{abstract}
Polymyalgia rheumatica (PMR) occurs almost exclusively in persons aged 50 years or older and it is the second most common inflammatory rheumatic disease in older people after rheumatoid arthritis. Since there are no specific tests for PMR, the exclusion of clinically similar differential diagnoses is essential to ascertain the diagnosis. These recommendations for the management of PMR assume an already established diagnosis of PMR. It is recommended to initiate
\end{abstract}

treatment with glucocorticoids immediately after diagnosis and to provide appropriate patient information and education about the impact of the disease and its treatment. Methotrexate should be considered in patients at high risk for relapse and/or glucocorticoidrelated adverse events. These guidelines have been elaborated because there is significant heterogeneity in the management of PMR in clinical practice in Germany (but also Europe and worldwide), despite the large number of patients with this disease. These guidelines are primarily based on the 2015 EULAR-ACR recommendations for the management of PMR, which were updated by the guideline committee and adapted to the German speaking countries.

\section{Keywords}

Polymyalgia rheumatica - Patient education as topic - Glucocorticoids · Methotrexate · EULARACR recommendations rameter der einzelnen Studien, wurde ebenso vom 2015 EULAR-ACR-Projekt übernommen und ist in - Tab. 2 dargestellt. Im ursprünglichen Projekt wurden die Outcome-Parameter in einem Teilprojekt definiert, bei welchem zuerst eine Liste an möglichen Parametern durch eine nichtsystematische Literatursuche und Experteninput erstellt wurde. Anschließend wurde diese Liste mithilfe einer Online-Umfrage unter Rheumatologen, Allgemeinmedizinern und Patienten verfeinert und schließlich von der Kommission zur Erstellung der 2015 EULAR-ACR-Leitlinien zur Abstimmung gebracht.

\section{Literatursuche}

Zur Erstellung der S3-Leitlinie wurde folgende Literatur herangezogen: (1) Studien, die in der SLR für die 2015 EULARACR-Empfehlungen identifiziert wurden, und (2) Arbeiten, die in einer Update-Suche für den Zeitraum von $04 / 2014$ bis $07 / 2016$ gefunden wurden. Die Methoden für die Update-Suche waren dabei identisch mit denen, die für die SLR der Quellleitlinie angewandt wurden.

Für die SLR erfolgte eine sensitive Suche zur Identifikation aller Artikel zum Thema PMR, die seit Januar 1970 publiziert wurden. Im 2015 EULAR-ACRProjekt wurde die SLR von Christian Dejaco (Graz, Österreich) und Yogesh Singh (Southend, Vereinigtes Königreich) durchgeführt. Die Update-Suche zur vorliegenden Leitlinie erfolgte durch Christian Dejaco (als R1 in - Abb. 1 bezeichnet) und Frank Buttgereit (Charité Berlin, Deutschland; R2 in • Abb. 1). Die SLR wurde jeweils von den 2 Gutach- 


\section{a) PICO-Fragen zu therapeutischen Interventionen}

1. In Polymyalgia rheumatica (PMR) (P), what is the effect of Nonsteroidal Anti-inflammatory drugs (NSAIDs) and/or analgesics (I) on outcome (O) compared with glucocorticoids (C)

2. In PMR (P), what is the effect of short duration of glucocorticoid therapy (I) on outcome (O) compared with long duration of glucocorticoid therapy (C)

3. In PMR (P), what is the effect of low dose oral glucocorticoids ( $\leq 7.5 \mathrm{mg} /$ day of prednisone equivalent) (I) on outcome (0) compared with medium dose of glucocorticoids $(>7.5 \mathrm{mg} /$ day but $\leq 30 \mathrm{mg} /$ day of prednisone equivalent) (C)

4. In PMR (P), what is the effect of medium dose oral glucocorticoids ( $>7.5 \mathrm{mg} /$ day but $\leq 30 \mathrm{mg} /$ day of prednisone equivalent) (I) on outcome $(0)$ compared with high dose of glucocorticoids ( $>30 \mathrm{mg} /$ day but $\leq 100 \mathrm{mg} /$ day of prednisone equivalent) (C)

5. In PMR (P), what is the effect of an oral glucocorticoid dose of $\geq 10 \mathrm{mg} /$ day but $\leq 20 \mathrm{mg} /$ day prednisone equivalent (I) on outcome (O) compared with a dose of $>20 \mathrm{mg}$ but $\leq 30 \mathrm{mg} /$ day of prednisone equivalent (C)

6. In PMR (P), what is the effect of rapid taper of glucocorticoids (I) on outcome (O) compared with slow taper of glucocorticoids $(C)$

7. In PMR (P), what is the effect of intramuscular injection of glucocorticoids (I) on outcome ( $\mathrm{O}$ ) compared with oral glucocorticoids (C)

\section{b) PICO-Fragen zu Prognosefaktoren}

13. In PMR (P), what is the effect of older age at diagnosis (I) on outcome $(0)$ compared with younger age $(C)$

14. In PMR (P), what is the effect of female sex (I) on outcome (O) compared with male sex (C)

15. In PMR (P), what is the effect of high levels of inflammatory markers (i. e. erythrocyte sedimentation rate [ESR] and/or C-reactive protein [CRP]) at diagnosis (I) on outcome $(\mathrm{O})$ compared with low levels of inflammatory markers (C)

16. In PMR (P), what is the effect of more active/severe disease at diagnosis (I) on outcome ( $\mathrm{O}$ ) compared with lower disease activity/ severity (C)

17. In PMR (P), what is the effect of the presence of peripheral arthritis at diagnosis $(\mathrm{I})$ on outcome $(\mathrm{O})$ compared with absence of peripheral arthritis (C)

18. In PMR (P), what is the effect of longer symptom duration at diagnosis (I) on outcome (O) compared with shorter symptom duration $(C)$

19. In PMR (P), what is the effect of concomitant conditions (including cardiovascular disease, cerebrovascular disease, peripheral vascular disease, osteoporosis, hyperlipidaemia, diabetes, hypertension, infection, cataract, glaucoma, peptic ulcer, skin disorders, adiposity, mood disturbances, cognitive disorder) at diagnosis that could be exaggerated by PMR and/or glucocorticoid therapy (I) on outcome (O) compared with absence of these conditions (C)

20. In PMR (P), what is the effect of rapid response to glucocorticoids (I) on outcome ( $\mathrm{O}$ ) compared with delayed response

21. In PMR (P), what is the effect of shared patients' management by primary and secondary care (I) on outcome $(0)$ compared to management in primary care only

22. In PMR (P), what is the effect of optimal control management of patients (I) on outcome $(\mathrm{O})$ compared to conventional management (C)

11. In PMR (P), what is the effect of biological agents (I) on outcome (O) compared with glucocorticoids alone (C)

12. In PMR (P), what is the effect of glucocorticoids plus non-pharmacological interventions (I) on outcome $(\mathrm{O})$ compared with glucocorticoids alone (C)

tern unabhängig durchgeführt, und im Falle einer fehlenden Übereinstimmung wurde der Konsens in der Diskussion gesucht. Konnte kein Konsens gefunden werden (dies betraf 15,6\% der Artikel im 2015 EULAR-ACR-Projekt, $0 \%$ in der Update-Suche) wurde ein weiterer Kollege (in der Quellleitlinie Andrew Hutchings, London, England) hinzugezogen.

\section{Suchstrategie und Datenbanken}

Für die SLR der Quellleitlinie und für die Update-Suche wurden identische Suchstrategien angewendet. In folgenden elektronischen Datenbanken wurde gesucht: Medline (Ovid), Embase, CINAHL, Web ofScience und Cochrane Library. Für jede Datenbank wurden als Schlüsselwörter die sog. „Thesauri“ (= Wortvorrat), Volltextwörter, verkürzte Wörter und Abkürzungen angewendet (s. - Tab. 3 für die Suchstrategie für Medline [Ovid]). Die „Grey Literatur“ (damit sind insbeson- dere publizierte Abstracts der Kongresse von EULAR, ACR, British Society of Rheumatology und von internationalen Kongressen mit den Themen PMR, Riesenzellarteriitis [RZA] und ANCA-assoziierte Vaskulitiden gemeint) und Studienregister wurden manuell mit dem Ziel durchsucht, weitere Vollpublikationen von relevanten Studien zu identifizieren. Zusätzlich wurden die Referenzlisten von Volltextartikeln durchsucht, und es wurden Experten zu möglichen weiteren Publikationen befragt. 
Tab. 2 Ergebnisparameter („outcome parameters"), welche für die systematische Literatursuche angewendet wurden

Disease remission

Disease relapse

Duration of glucocorticoid therapy

Discontinuation of glucocorticoid therapy

Development of giant cell arteritis

Glucocorticoid side effects (diabetes mellitus/glucose intolerance, osteoporosis, cardiovascular disease, dyslipidemia, impaired wound healing, infections, osteonecrosis, myopathy, cataract, glaucoma, atherosclerosis, hypertension, peptic ulcer, weight gain, moon face, dyspnea, palpitations, fatigue, skin atrophy, bruising, mood disorders)

Response to glucocorticoid therapy

Cumulative glucocorticoid dose

Acute phase reactants

Patients assessment of global wellbeing

Severity/duration of morning stiffness

Lowest possible glucocorticoid dose (prednisone equivalent less than $5 \mathrm{mg} /$ day)

Functional status (Health Assessment Questionnaire or other measures)

Quality of life (Short Form-36, EQ5D etc.)

Mortality

Hospitalization (due to disease, its complications, co-morbidity and/or treatment related complications)

Impact on patients' social environment

Fatigue

Imaging of shoulder/hip

Healthcare resource use (health economics)

Disease activity score

\section{Ein- und Ausschluss von Studien, Datenmanagement}

Die Zitate und Abstracts der identifizierten Artikel wurden in eine BibliografieSoftware (Zotero Version 4.0.20, Fairfax, VA, USA) übertragen, Duplikate wurden mithilfe der Software entfernt. Danach wurde ein Screening der Titel und Abstracts durchgeführt, um nichtrelevante Literatur zu entfernen. Die verbleibenden Artikel wurden im Volltext durchgesehen und hinsichtlich der Ein- und Ausschlusskriterien überprüft.

Eingeschlossen wurden nur solche Artikel, die für ein oder mehrere PICOFragen relevant waren. Alle Studien, die keine Originaldaten berichteten, Patienten untersuchten, die nicht an einer PMR litten, oder Patienten mit PMR und RZA als eine einzige Gruppe untersuchten, wurden ausgeschlossen. Zudem wurden alle Studien $\mathrm{zu}$ Prognosefaktoren ausgeschlossen, wenn sie Parameter untersuchten, die nicht in der klinischen Routine verfügbar waren. Alle relevanten Studiendetails der eingeschlossenen Studien wurden in einer vorgefertigten Extraktionstabelle („data extraction sheet") aufgelistet.

\section{Qualitätsbeurteilung}

Sowohl in der Quellleitlinie als auch beim Update-SLR wurde die Qualität der Studien $\mathrm{zu}$ Interventionen mithilfe der GRADE-Methode und die Qualität derStudien zu Prognosefaktoren mit dem Quality-In-Prognosis-Studies(QUIPS)Tool beurteilt. Bei GRADE wird die Qualität der gesamten Evidenz über mehrere Studien hinweg für jeden Outcomeparameter beurteilt. Es ist daher möglich, dass eine Studie, die mehrere Outcomes adressiert, für jedes der Outcomes unterschiedliche Evidenzqualitäten aufweist. GRADE teilt die Qualität der Evidenz in folgende Stufen ein: hoch, moderat, niedrig, sehr niedrig. Folgende Domänen werden dabei zur Beurteilung der Evidenzqualität (LoE) herangezogen: (1) Studienlimitationen (Limitationen bei der Randomisierung, Geheimhaltung der Gruppenzuordnung,
Tab. 3 Schlüsselwörter für die Literatur-

suche in Medline (Ovid)

1. Polymyalgia rheumatica \#

2. Polymyalgia rheumatica $\$$

3. polymyalgia rheumatica. $\mathrm{mp}$

4. PMR NOT prenatal mortality rate $\$ . m p$

5. PMR NOT premature mortality rate $\$ . m p$

6. PMR NOT population mortality rate $\$ . \mathrm{mp}$

7. polymyalgi*

8. polymyalgia $\$ . m p$

9. rheumatic polymyalgia \$.mp

10. polymyalgia arteritica $\$ . m p$

11. forestier certonciny syndrome $\$ . \mathrm{mp}$

12. pseudopolyarthritis rhizomelica $\$ . \mathrm{mp}$

13. rheumatic myalgia $\$ . m p$

14. rheumatism, inflammatory rhizomelic $\$$ .mp

15. rhizomelic pseudopolyarthritis $\$ . m p$

*truncation; \# MeSH term; \$ textword;

$\mathrm{mp}=$ title, abstract, subject headings, head-

ing word, drug trade name, original title,

device manufacturer, drug manufacturer,

device trade name, keyword

Verblindung der Intervention, Anzahl der Patienten, die die Studie nicht abgeschlossen haben, Nichtanwendung des Intention-to-Treat-Prinzips, vorzeitiger Studienabbruch und selektives Berichten von Outcomes), (2) Inkonsistenz der Ergebnisse über mehrere Studien hinweg, (3) indirekte Evidenz (indem Population, Intervention und/oder Outcome in den Studien nicht oder nur teilweise denen der PICO-Frage entsprechen), (4) fehlende Präzision der Ergebnisse und (5) Publikationsbias. Randomisierte Studien starten mit hoher Evidenzqualität, können aber im Falle von Limitationen um je 1 bis 2 Grad(e) niedriger eingestuft werden. Nicht randomisierte Studien starten hingegen bereits mit einer niedrigen Qualität. Unter bestimmten Umständen ist allerdings auch ein Höherstufen der Evidenzqualität möglich [8]. Beim QUIPS-Tool wird der mögliche Bias (bewertet als hohe, moderate oder niedrige Gefahr für Bias) für jede der folgenden Kategorien (insgesamt 8 Kategorien, wobei die letzten beiden dem originalen QUIPS-Tool hinzugefügt wurden) einzeln bewertet: (1) Auswahl der Studienteilnehmer, (2) Zahl und Eigenschaften der Teilnehmer, welche die Studie 


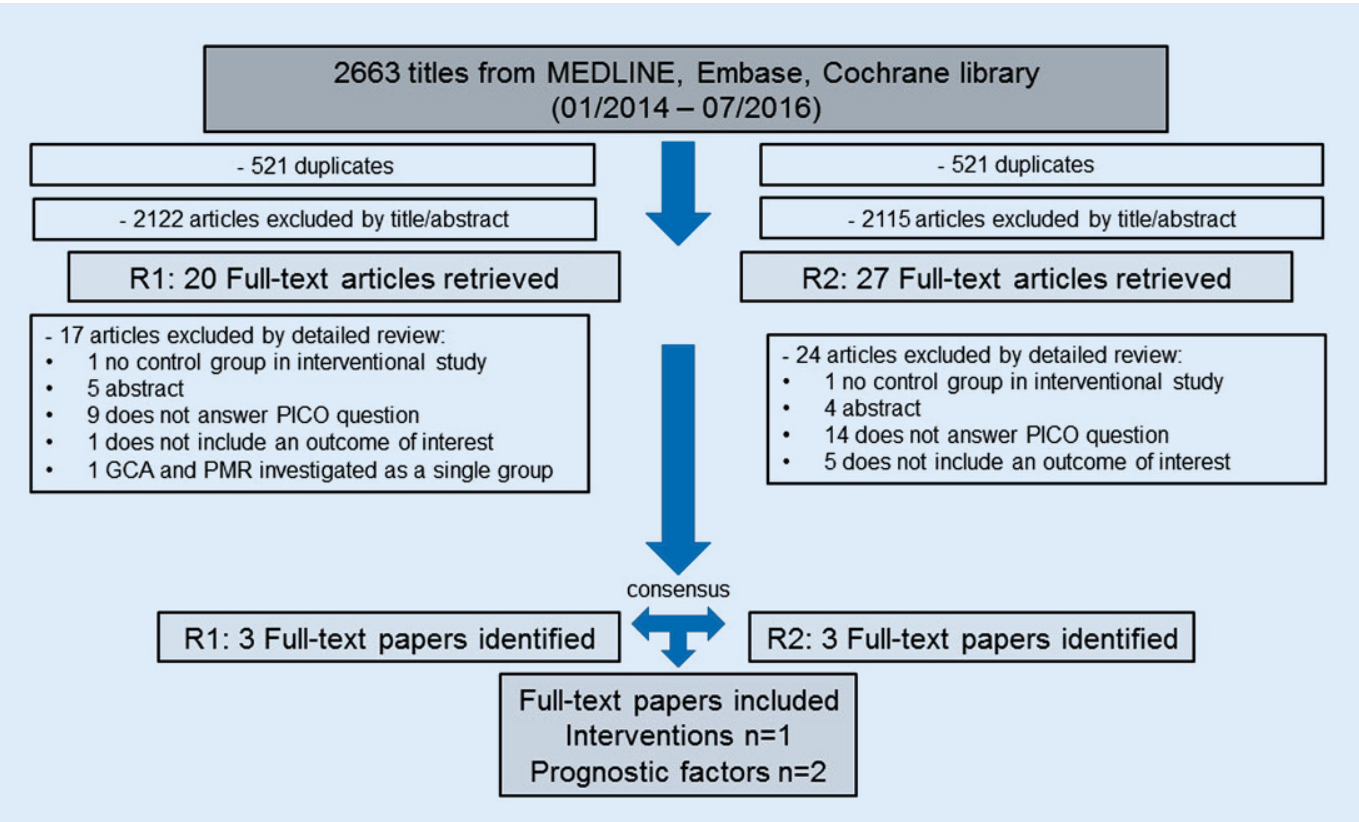

Abb. $1<$ Aktualisierung der systematischen Literatursuche für den Zeitraum Januar 2014 bis Juli 2016

nicht abgeschlossen haben, (3) Messung des Prognosefaktors, (4) Berücksichtigung von Störvariablen, (5) Messung der Outcomes, (6) statistische Analyse, (7) die mögliche Beeinflussung durch Einschluss von RZA-Patienten und (8) andere Möglichkeiten von Bias. Im Ergebnisteil wird die Anzahl der Kategorien mit einem niedrigen Risiko für Bias („Low risk of Bias“ [LoB]) dargestellt.

Wann immer möglich und sinnvoll, wurde v. a. bei Interventionsstudien versucht, eine Metaanalyse durchzuführen. In allen anderen Fällen wurden die Ergebnisse für jede Studie einzeln präsentiert.

Der Leitlinienkommission wurde die Evidenzqualität nach GRADEvorgestellt. Um dem Leser einen besseren Überblick über die Evidenzlage zu ermöglichen und auch den Richtlinien für die Präsentation von S3-Leitlinien folgend, wurde zusätzlich der Evidenzgrad der einzelnen Empfehlungen anhand des $\mathrm{Ox}$ ford Centre for Evidence-Based Medicine (OCEBM) bestimmt (http://www. cebm.net/index.aspx?o=5653). Diese Bewertung dient nur zur Information des Lesers und spielte bei der Erstellung der Leitlinien keine Rolle.

Anhand des OCEBM wird der Evidenzgrad für Interventionsstudien in 5 Stufen eingeteilt: Level 1 - systematischer Review von mehreren rando- misierten Studien, Level 2 - einzelne randomisierte Studie mit ausgeprägtem Effekt, Level 3 - nicht randomisierte Studie, Level 4 - Fallserien, Case-control-Studie oder Studie mit historischen Kontrollen, Level 5 - Expertenmeinung.

\section{Formulierung der Empfehlungen}

Für die Formulierung der Empfehlungen wurde ein nominaler Gruppenprozess angewendet. Als Grundlage für die Diskussion dienten die ins Deutsche übersetzten Empfehlungen der 2015 EULAR-ACR Recommendations. Die Übersetzung wurde von Frank Buttgereit (Charité Berlin, Deutschland) durchgeführt. Zudem wurden bei der Sitzung der Leitlinienkommission im Februar 2017 die gesamte Evidenz und deren Qualität präsentiert. Dazu gehörten alle Studien, die im Rahmen des 2015 EULAR-ACR-Projekts identifiziert wurden, sowie alle in der Update-SLR identifizierten Artikel.

Für die Formulierung der Empfehlungen anhand von GRADE sollten durch die Leitlinienkommission folgende Aspekte berücksichtigt werden: (1) Qualität der Evidenz, (2) Verhältnis zwischen erwartetem Nutzen und Risiko einer Intervention, (3) angenommene Werte und Präferenzen der Patienten und (4) Ressourcenverbrauch. Die Daten zu den Pro- gnosefaktoren wurden zur Bildung von Subgruppen angewendet, für welche die Empfehlungen in Folge genauer zugeschnitten wurden.

Im Unterschied zu GRADE, wonach der Grad einer Empfehlung „stark“ oder "bedingt" "dafür“ oder „dagegen“ sein kann, einigte sich die Leitlinienkommission dieser S3-Leitlinie auf das AB0-System bezüglich der Stärke von Empfehlungen. Dabei wurde für A (starke Empfehlung) die Formulierung „soll“, für B (bedingte Empfehlung) die Formulierung „sollte oder kann“ und für 0 die Aussage „Dazu kann keine Empfehlung abgegeben werden" verwendet.

Nach Diskussion und Modifikation der vorgeschlagenen Empfehlungen für die S3-Leitlinie wurde von der Leitlinienkommission über die Annahme der Empfehlungen abgestimmt, wobei eine Zustimmung von zumindest $75 \%$ aller stimmberechtigten Mitglieder der Leitlinienkommission zum Endprodukt notwendig war.

Wie in den 2015 ACR-EULAR-Empfehlungen wurden auch für die S3Leitlinie sog. „übergeordnete Prinzipien“ vorangestellt (eng.: „overarching principles“), die nach dem Verständnis der Leitlinienkommission dem aktuellen Betreuungsstandard in der Behandlung der PMR entsprechen und somit nicht direkt auf den Ergebnissen der SLR be- 
ruhen. Die Leitlinienkommission war der Ansicht, dass diese übergeordneten Prinzipien die "gute klinische Praxis“ widerspiegeln und es daher ethisch nicht vertretbar wäre, diese Prinzipien in einer placebokontrollierten Studie zu untersuchen.

Nach der zweiten Sitzung der Leitlinienkommission im Februar 2017 wurden die konsentierten und sprachlich optimierten Empfehlungen der S3-Leitlinie nochmals via E-Mail an alle Kommissionmitglieder mit der Bitte um Kommentierung oder finale Zustimmung zugesandt. Das fertige Manuskript wurde mit allen beteiligten Fachgesellschaften abgestimmt.

\section{Ergebnisse}

Die für die 2015 EULAR-ACR-Empfehlung identifizierten (und für diese Arbeit herangezogenen Artikel) waren wie folgt: 16 Artikel zu therapeutischen Interventionen, $30 \mathrm{zu}$ Prognosefaktoren und 6 zu Arbeiten, bei denen sowohl eine Intervention als auch ein prognostischer Faktor untersucht wurden [9].

Das Ergebnis der Update-Literatursuche ist in - Abb. 1 dargestellt.

Insgesamt wurden 3 Artikel (1 zu therapeutischen Interventionen und 2 zu Prognosefaktoren) durch die UpdateSLR identifiziert (- Tab. 4 und 5). Eine offene Studie untersuchte die Wirksamkeit von Tocilizumab an 10 PMR-Patienten, und 10 Patienten, die für die Teilnahme an der Studie nicht geeignet waren, dienten als Kontrollen [10]. Eine Glukokortikoid-freie Remission nach 6 Monaten wurde bei $100 \%$ der Patienten der Interventionsgruppe, allerdings bei keinem der Kontrollgruppe erreicht (LoE niedrig). Zumindest 1 Rezidiv innerhalb von 6 Monaten trat bei keinem Patienten in der Tocilizumab-Gruppe, hingegen bei $60 \%$ in der Kontrollgruppe auf (LoE sehr niedrig). Die kumulative Glukokortikoid-Dosis (1,1 vs. 2,6g, LoE niedrig) und die Dauer der Glukokortikoid-Therapie (3,9 vs. 14,1 Monate) waren in der Tocilizumab-Gruppe niedriger bzw. kürzer [10]. Die beiden anderen Studien beschäftigten sich mit dem Wert von Prognosefaktoren. Höheres Alter (LoB 2/8) und männliches Geschlecht (LoB
2/8) wurde in einer Studie mit $3249 \mathrm{~Pa}$ tienten mit einem erhöhten Risiko an kardiovaskulären Ereignissen assoziiert [11]. In einer anderen Studie wurde in Bezug auf das Ansprechen einer Glukokortikoid-Therapie kein Unterschied zwischen männlichem und weiblichem Geschlecht (LoB 6/8) und kein Unterschied zwischen Patienten mit und ohne periphere Arthritis (LoB 5/8) beobachtet [12].

Die final konsentierten übergeordneten Prinzipien und die spezifischen Empfehlungen zur Behandlung von Patienten mit PMR sind in - Tab. 6 und 7 dargestellt.

\section{Diskussion}

Diese Leitlinie richtet sich in erster Linie an Rheumatologen/-innen, weil die Erkrankung PMR in dieses Fachgebiet gehört und Patienten mit PMR daher primär internistisch-rheumatologisch betreut werden sollten. Viele Patienten (mit unkomplizierter PMR) werden jedoch auch durch Ärzte/Ärztinnen anderer Fachrichtungen (z. B. hausärztlich und fachärztlich tätige Internisten, Fachärzte für Allgemeinmedizin, Orthopädie, Geriatrie oder physikalische und rehabilitative Medizin) behandelt, weswegen diese Leitlinie auch diese Fachgruppen sowie Angehörige nichtärztlicher Berufsgruppen anspricht, die sich direkt oder indirekt an der Betreuung von Patienten mit PMR beteiligen. Mit dem übergeordneten Prinzip C wird jedoch unterstrichen, dass PMR-Patienten mit atypischer Klinik, komplizierten Verläufen oder häufigen Rezidiven üblicherweise durch einen spezialisierten Rheumatologen betreut werden sollen.

Übergeordnete Prinzipien. Diese Leitlinie geht davon aus, dass Erkrankungen mit PMR-ähnlichen Symptomen bereits ausgeschlossen worden sind. In diesem Zusammenhang wird in den übergeordneten Prinzipien für die Behandlung von Patienten mit PMR darauf hingewiesen, dass vor Einleitung der Therapie geeignete Labor- und/oder apparative Untersuchungen durchzuführen sind. Die Details dazu sind in den 2015 EULAR-ACR-Empfehlungen zum Management der Polymyalgia rheu- matica (die als Quellleitlinie verwendet wurde) genannt und sind daher hier übernommen worden. Zusätzliche Informationen dazu sind in einem Review aus dem Jahr 2016 nachzulesen [3]. Die Leitlinienkommission schließt sich ebenfalls vollumfänglich den EULARACR-Empfehlungen an, wonach bei jedem Patienten individuell existierende Komorbiditäten und Komedikationen sowie mögliche Risikofaktoren für einen ungünstigen Krankheitsverlauf (häufige Rezidive, verlängerte Behandlungsdauer mit und/oder Nebenwirkungen durch Glukokortikoide) berücksichtigt werden müssen. Patienten mit atypischen Zeichen und Symptomen, Auftreten oder hohem Risiko von bzw. für therapiebezogene(n) Nebenwirkungen, mit einer gegenüber der Glukokortikoid-Therapie refraktären PMR und/oder mit wiederholten Rezidiven (Rezidiv: Wiederkehr der Symptome der PMR und Anstieg der Entzündungsparameter) und/oder der Notwendigkeit zu einer sehr langen Therapie werden üblicherweise durch einen Spezialisten behandelt.

Es wird kontrovers diskutiert, ob ein Zusammenhang zwischen PMR und Tumorerkrankungen besteht [13]. Während einige longitudinale Studien keine Assoziation der PMR mit Malignomen zeigen konnten [14, 15], berichteten Haugeberg et al. über eine „erhöhte Frequenz von Tumorerkrankungen bei Patienten mit neu aufgetretenen polymyalgischen Symptomen, bei denen eine PMR vermutet wurde" [16]. Daher ist die Möglichkeit in Betracht zu ziehen, dass eine Paraneoplasiesymptomatik eine PMR vortäuscht. Die LLKommission hat dieser Tatsache mit der Formulierung des übergeordneten Prinzips A Rechnung getragen, wonach selbst bei Vorliegen einer klinisch hinreichenden Befundkonstellation Erkrankungen mit PMR-ähnlichen Symptomen (z.B. nichtentzündliche, entzündliche, medikamenteninduzierte, endokrine, infektiöse oder neoplastische Erkrankungen) ausgeschlossen werden sollten. Daraus kann jedoch keine Empfehlung zu einem generellen oder spezifischen, über das altersentsprechende, übliche Maß hinausgehenden Tumorscreening abgeleitet werden. Von möglicher klinischer 


\section{Leitlinien}

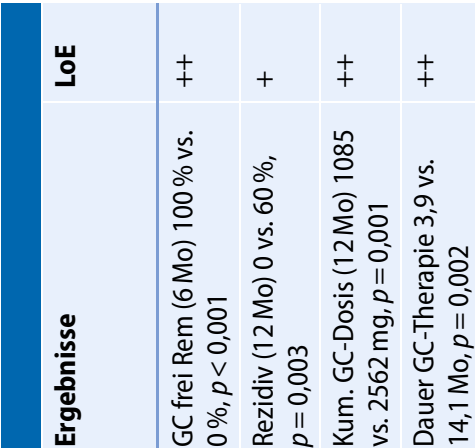

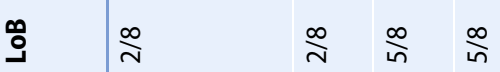

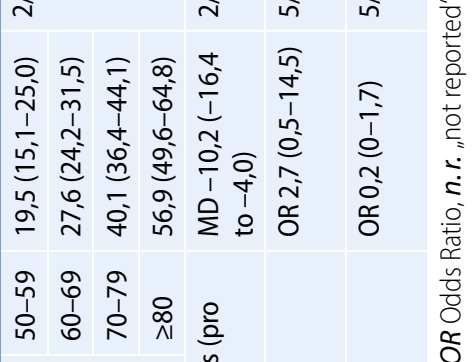

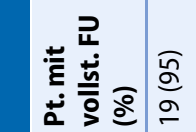

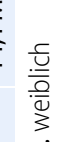

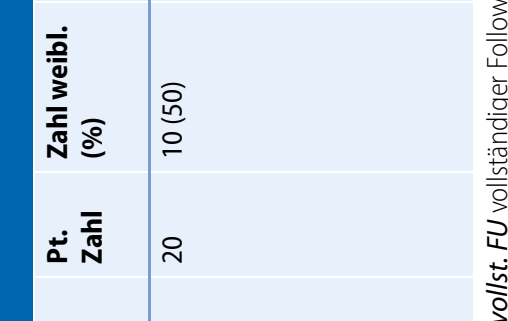

을

童

돌

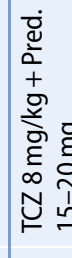

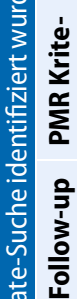

졿

\section{然}

西

गัฐ

高

产

क्ष

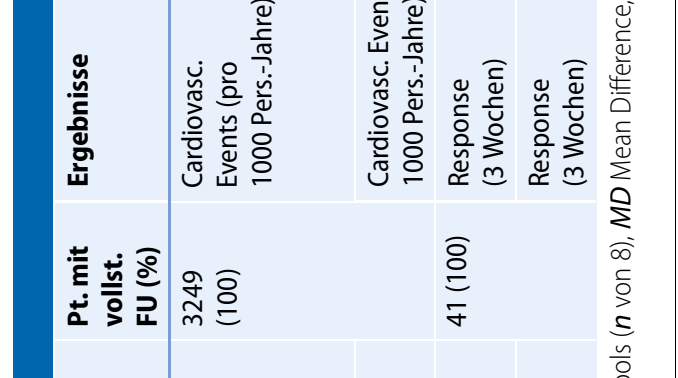

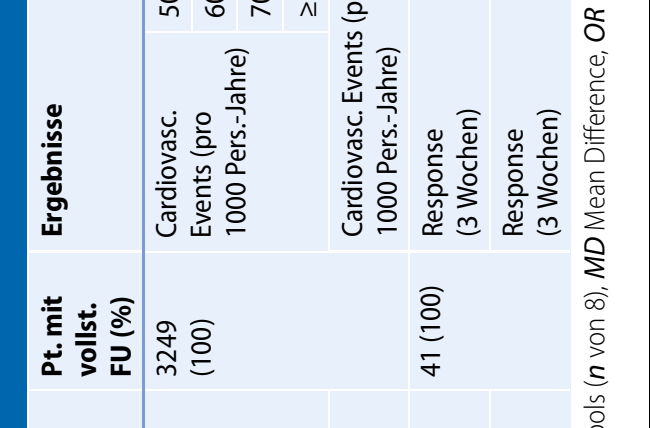

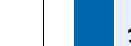

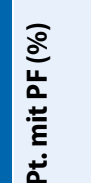

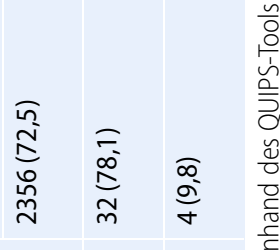

迹

离

ह

窇离离

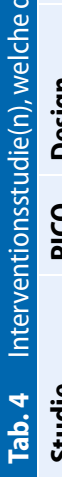

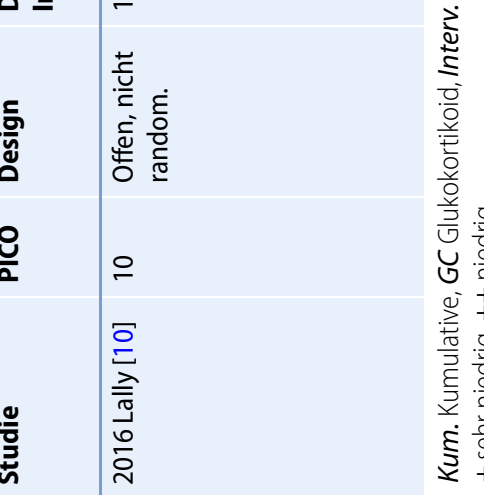

$\frac{8}{0}$

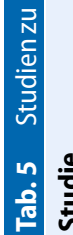
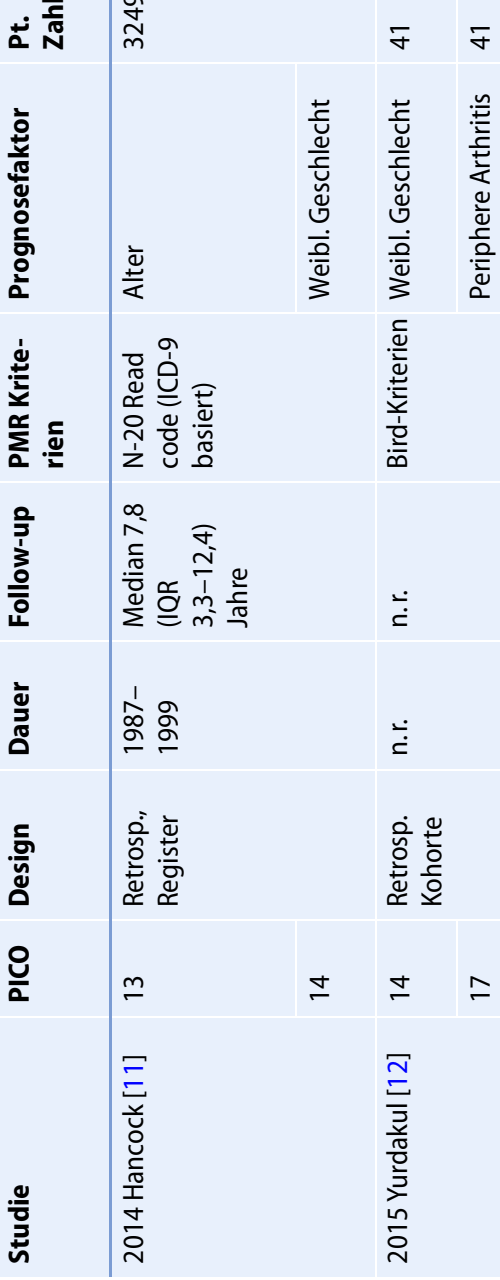

范

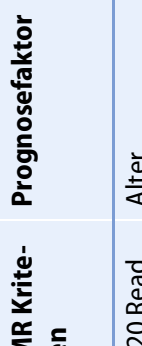

离

o

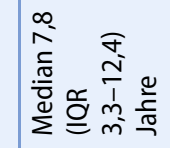

?

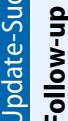

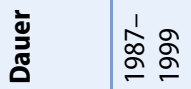

$\stackrel{\dot{S}}{\dot{2}}$

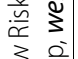

곤

E 
Tab. 6 Übergeordnete Prinzipien für die Behandlung von Patienten mit Polymyalgia rheumatica

A. Selbst bei Vorliegen einer klinisch hinreichenden Befundkonstellation sollten Erkrankungen mit PMR-ähnlichen Symptomen (z. B. nichtentzündliche, entzündliche, medikamenteninduzierte, endokrine, infektiöse oder neoplastische Erkrankungen) ausgeschlossen werden

B. Bei jedem Patienten mit PMR gehört es zur guten medizinischen Praxis, vor Einleitung der Therapie geeignete Labor- und/oder apparative Untersuchungen durchzuführen

In Abhängigkeit von den klinischen Zeichen und Symptomen sowie der Wahrscheinlichkeit, dass eine andere Diagnose vorliegt, können die folgenden Untersuchungen durchgeführt werden:

- Laborparameter:

Rheumafaktor, Anti-CCP-Antikörper, CRP, BSG, Serumelektrophorese, Blutbild, Glukose, Kreatinin, Leberfunktionsparameter, Kalzium, alkalische Phosphatase, Vitamin D, Urin-Stick-Analyse, TSH, CK, ANA, ANCA oder Tuberkulosetests

- Apparative Untersuchungen:

Röntgen-Thorax, Abdomensonographie, Messung der Knochendichte Bei der Behandlung eines jeden Patienten mit PMR gehört zur guten medizinischen Praxis, dass existierende Komorbiditäten und Komedikationen berücksichtigt werden. Dazu gehören u. a. die arterielle Hypertonie, Diabetes mellitus, gestörte Glukosetoleranz, kardiovaskuläre Erkrankungen, Dyslipidämien, peptisches Ulkus, Osteoporose, Katarakt, Glaukom, Infektionen und eine Behandlung mit nichtsteroidalen Antirheumatika

Die Bedeutung von Risikofaktoren für einen ungünstigen Krankheitsverlauf ist bislang noch nicht klar. Mögliche Risikofaktoren für eine höhere Rezidivrate und/oder eine verlängerte Behandlungsdauer sind: weibliches Geschlecht, hohe BSG ( $>40 \mathrm{~mm} / \mathrm{h}$ ) und periphere Arthritis

C. Patienten mit atypischen Zeichen und Symptomen (z. B. periphere Arthritis, systemische Symptome, niedrige Entzündungsparameter, Alter $<60$ Jahre), hohem Risiko für oder Auftreten von therapiebezogene(n) Nebenwirkungen, mit einer gegenüber der Glukokortikoid-Therapie refraktären PMR und/oder mit wiederholten Rezidiven und/oder der Notwendigkeit zu einer sehr langen Therapie werden üblicherweise durch einen Spezialisten behandelt

D. Die Behandlung von PMR-Patienten hat die bestmögliche Versorgung zum Ziel und basiert auf einer gemeinsamen Entscheidung von Patient und behandelndem Arzt. Dies setzt eine angemessene Patientenschulung in Bezug auf die Auswirkungen der PMR und deren Behandlung voraus

E. Folgevisiten werden durchgeführt:

- Alle 4 bis 8 Wochen im ersten Jahr

- Alle 8 bis 12 Wochen im zweiten Jahr sowie bei Rezidiven und bei Nebenwirkungen

- Bei Bedarf nach Absetzen der medikamentösen Therapie

Bei jeder Folgevisite werden folgende klinische und laborchemische Parameter erfasst: Krankheitsaktivität, (Risikofaktoren für) Nebenwirkungen der Therapie, Begleiterkrankungen und Begleitmedikation, Rezidive und Behandlungsdauer

"Evidence Levels nach dem Oxford Centre for Evidence-Based Medicine (OCEBM). Diese Bewertung erfolgte, nachdem über die Empfehlungen abgestimmt wurde, und dient zur Information des Lesers ** Der Grad der Zustimmung sollte mit einer Zahl zwischen 0 und 10 angegeben werden, wobei die Eckwerte 0 als "keine Zustimmung" bzw. 10 als "maximale Zustimmung" definiert wurden
Bedeutung sind die älteren Beobachtungen von Naschitz et al., wonach das Vorliegen einer paraneoplastischen PMR-Symptomatik in Betracht gezogen werden sollte bei Patienten mit sehr ausgeprägten konstitutionellen Symptomen in einem Alter von $<50$ Jahren, Asymmetrie der Symptome, fehlender Effektivität von Prednison, BSG von $<40$ oder $>100 \mathrm{~mm} / \mathrm{h}$ und einer peripheren Arthritis [17, 18]. Davon abweichend, haben Bellan et al. kürzlich als die stärksten Prädiktoren für eine paraneoplastische PMR die folgenden publiziert: 6 oder mehr schmerzhafte Gelenke, Alter >75 Jahre und männliches Geschlecht [13].

Von zentraler Bedeutung für den Behandlungserfolg sind Therapieentscheidungen, die gemeinsam durch Patient und behandelnden Arzt initial und anlässlich von Folgevisiten in geeigneten Intervallen getroffen werden, sowie eine angemessene Patientenschulung in Bezug auf die Auswirkungen der PMR (u. a. Einschränkung der Lebensqualität durch die Symptomatik, potenzielles Auftreten einer Riesenzellarteriitis) und deren Behandlung (insbesondere das Nutzen-Risiko-Verhältnis der Therapie mit Glukokortikoiden).

Empfehlungen. Im Ergebnis der Arbeit der Leitliniengruppe sind die folgenden Empfehlungen erarbeitet und konsentiert worden. Mit Level* wird der jeweilige Evidence Level nach dem Oxford Centre for Evidence-Based Medicine (OCEBM) angegeben.

Empfehlung 1. Bei Patienten mit PMR soll unmittelbar nach Diagnosestellung die Therapie mit Glukokortikoiden eingeleitet werden. (Level ${ }^{*} 5$ )

Bei der Behandlung der PMR sind die Glukokortikoide (GC) nach wie vor Mittel der ersten Wahl, weil diese Behandlung bei den meisten Patienten eine rasche und deutlich ausgeprägte Linderung der Beschwerden bewirkt. Daher ist die Anwendung von GC in der Praxis sehr gut etabliert [1]. Nichtsteroidale Antirheumatika sollen nicht zur Therapie der PMR eingesetzt werden, weil das potenzielle Risiko für Nebenwirkungen größer ist als der zu erwartende, meist ge- 
Tab. 7 Spezifische Empfehlungen zur Behandlung von Patienten mit Polymyalgia rheumatica

Spezifische Empfehlung

Grad der Zu-

stimmung**

1. Bei Patienten mit PMR soll unmittelbar nach Diagnosestellung die

10,00

Therapie mit Glukokortikoiden eingeleitet werden. (Level*5)

2. Die Dosierung der Glukokortikoid-Therapie soll für jeden PMR-Patienten individuell angepasst werden. Sie sollte immer so hoch wie nötig, aber so niedrig wie möglich sein. (Level* 5 )

2a. Art der Glukokortikoid-Anwendung:

Glukokortikoide sollten bei der Behandlung der PMR oral angewendet werden. (Level ${ }^{*}$ 5)

2b. Zeitpunkt der Anwendung:

Glukokortikoide sollten in einer morgendlichen Einzeldosis gegeben werden. (Level* 5)

2c. Initialdosis:

Die Glukokortikoid-Initialdosis sollte bei den meisten Patienten mit PMR zwischen 15 und 25 mg Prednison-Äquivalent pro Tag liegen. Es sollen keine Initialdosen von $\leq 7,5 \mathrm{mg} / \mathrm{Tag}$ oder von $>30 \mathrm{mg} / \mathrm{Tag}$ angewendet werden. (Level* 2 )

2d. Reduktion bzw. Anpassung:

Die Glukokortikoid-Dosis soll kontinuierlich reduziert werden, basierend auf einem regelmäßigen Monitoring der Krankheitsaktivität des Patienten, der Laborparameter und des Auftretens von Nebenwirkungen

Die folgenden Prinzipien zur Reduktion bzw. Anpassung der Glukokortikoid-Dosis werden empfohlen:

- Bei der initialen Reduktion sollte eine orale Dosis von $10 \mathrm{mg} / \mathrm{Tag}$

Prednison-Äquivalent innerhalb von 4 bis 8 Wochen erreicht werden

- Danach sollte die tägliche orale Prednison-Dosis um etwa $1 \mathrm{mg}$ alle 4 Wochen bis zum Absetzen reduziert werden

Wird während des Absenkens der Glukokortikoid-Therapie die Krankheit wieder aktiv (Rezidiv), sollte die orale Prednison-Dosis zumindest auf die Prä-Rezidiv-Dosis erhöht und dann schrittweise innerhalb von 4 bis 8 Wochen wieder reduziert werden auf die Dosis, bei der das Rezidiv auftrat. (Level* 5)

2e. Behandlungsdauer:

Die Dauer der Glukokortikoid-Therapie soll für jeden PMR-Patienten individuell angepasst werden. Die Behandlungsdauer sollte so lang wie nötig, aber so kurz wie möglich sein. (Level* 5)

3. Zusätzlich zur Glukokortikoid-Therapie sollte die Gabe von Methotrexat frühzeitig in Betracht gezogen werden, insbesondere bei Patienten mit hohem Risiko für Rezidive und/oder für eine lange Therapiedauer sowie bei Patienten mit Risikofaktoren, Komorbiditäten und/oder Begleitmedikationen, bei denen Glukokortikoid-induzierte Nebenwirkungen mit höherer Wahrscheinlichkeit auftreten. Methotrexat kann auch erwogen werden bei Patienten mit Rezidiv(en), unzureichendem Ansprechen auf Glukokortikoide oder bei Auftreten Glukokortikoid-induzierter Nebenwirkungen. (Level* ${ }^{*}$ )

4. PMR-Patienten sollen nicht mit TNF-a-blockierenden Substanzen behandelt werden. (Level* 1)

Zu anderen Biologika inklusive Tocilizumab kann derzeit keine Empfehlung abgegeben werden

5. Insbesondere älteren und/oder gebrechlichen Patienten sollte zusätzlich zur medikamentösen Therapie ein individualisiertes Übungsprogramm angeboten werden. (Level* 5)

"Evidence Levels nach dem Oxford Centre for Evidence-Based Medicine (OCEBM). Diese Bewertung erfolgte, nachdem über die Empfehlungen abgestimmt wurde, und dient zur Information des Lesers

*** Der Grad der Zustimmung sollte mit einer Zahl zwischen 0 und 10 angegeben werden, wobei die Eckwerte 0 als „keine Zustimmung" bzw. 10 als „maximale Zustimmung" definiert wurden ringe therapeutische Nutzen. NSAR und/ oder Analgetika können jedoch zusätzlich angewendet werden, wenn Schmerzen anderer Ursachen bestehen. Es können keine spezifischen Empfehlungen zu Analgetika gegeben werden.

Empfehlung 2. Die Dosierung der Glukokortikoid-Therapie soll für jeden PMRPatienten individuell angepasst werden. Sie sollte immer so hoch wie nötig, aber so niedrig wie möglich sein. (Level ${ }^{*} 5$ )

Eine spezifischere Empfehlung kann nicht gegeben werden, weil es zu wenig Publikationen von hoher Evidenz zu dieser Frage gibt und weil zu viele Subgruppen von Patienten mit unterschiedlichem klinischem Profil existieren. Für die Quellleitlinie kam die Autorengruppe daher zu dem Konsensus, dass nur eine individualisierte, d.h. patientenspezifische Behandlung Mittel der Wahl sein kann (s. auch Empfehlung 2c und 2d). Damit ist gemeint, dass diese Therapie einerseits effektiv sein muss, andererseits jedoch Mindestwerte angestrebt werden sollen, was Dosis und Dauer der Behandlung mit GC betrifft. Dabei müssen patientenspezifische Risikofaktoren für das Auftreten von GC-induzierten Nebenwirkungen, Erkrankungsrezidiven und die Notwendigkeit zu einer Langzeitbehandlung ebenso wie Fragen nach dem Vorliegen von Komorbiditäten und Begleitmedikationen in die Therapieentscheidung einbezogen werden. Nur so kann ein bestmögliches Nutzen-Risiko-Verhältnis erreicht werden.

Empfehlung 2a (Art der Anwendung). Glukokortikoide sollten bei der Behandlung der PMR oral angewandt werden. (Level $\left.{ }^{\star} 5\right)$

Im deutschsprachigen Raum wird die orale Anwendung von GC bevorzugt. Allerdings kann Methylprednisolon intramuskulär als eine Alternative zu oralen Glukokortikoiden in Betracht gezogen werden. Die Wahl zwischen oraler Glukokortikoid-Therapie und intramuskulärer Methylprednisolon-Anwendung unterliegt der Entscheidung des behandelnden Arztes. In einer erfolgreichen klinischen Studie wurden initial $120 \mathrm{mg}$ Methylprednisolon alle 3 Wochen gegeben [19]. In Deutschland, Österreich und 
der Schweiz (und anderen Ländern) ist jedoch die intramuskuläre Anwendung von Methylprednisolon bei der PMR unüblich.

\section{Empfehlung 2b (Zeitpunkt der Anwen-}

dung). Glukokortikoide sollten in einer morgendlichen Einzeldosis gegeben werden. $\left(\right.$ Level $\left.^{\star} 5\right)$

Es gibt keine Studien, die die Frage nach dem Zeitpunkt der Anwendung von konventionellen GC spezifisch adressiert hat. Basierend auf guten klinischen Erfahrungen mit der Einzelgabe und wegen der Befürchtung, dass Nebenwirkungen (z.B. eine Hypothalamus-HypophysenNebennierenrinden-Insuffizienz, Beeinträchtigungen der zirkadianen Rhythmik, Schlafstörungen) häufiger auftreten können, haben sich die Autoren der Quellleitlinie gegen die generelle Anwendung einer geteilten täglichen GC-Dosis bei der PMR ausgesprochen. Dagegen kann in speziellen Situationen sehr wohl die Dosis geteilt werden, z. B. wenn nächtliche Schmerzen während der Reduktion der GC-Dosis im Bereich unterhalb von $5 \mathrm{mg} / \mathrm{Tag}$ PrednisonÄquivalent auftreten.

Kürzlich wurde eine Studie publiziert, die Effektivität und Sicherheit von „modified-release" (MR) Prednison bei Patienten mit neu diagnostizierter PMR untersuchte [20]. Obwohl infolge von Rekrutierungsschwierigkeiten nur 62 Patienten (400 waren geplant) eingeschlossen wurden, war der Anteil von Patienten mit vollständigem Ansprechen zu Woche 4 in der MR-Gruppe numerisch höher $(53,8 \%)$ als in der Vergleichsgruppe, die mit konventionellem Prednison behandelt wurde (40,9\%). Diese Evidenzlage rechtfertigt noch nicht den Eingang in Empfehlungen.

Empfehlung 2c (Initialdosis). Die Glukokortikoid-Initialdosis sollte bei den meisten Patienten mit PMR zwischen 15 und $25 \mathrm{mg}$ Prednison-Äquivalent pro Tag liegen. Es sollen keine Initialdosen von $\leq 7,5 \mathrm{mg} / \mathrm{Tag}$ oder von $>30 \mathrm{mg} / \mathrm{Tag}$ angewandt werden. (Level ${ }^{*} 2$ )

Der empfohlene Bereich für die Initialdosis ist deshalb so breit, weil es sehr viele verschiedene Subgruppen von PMR-Patienten gibt, die sich bezüglich des Risikos für ein Rezidiv, die Notwendigkeit für eine lange Behandlungsdauer und/oder das Auftreten von GC-induzierten Nebenwirkungen sowie bezüglich der Komorbiditäten und Begleitmedikation teilweise erheblich voneinander unterscheiden. Daher können keine evidenzbasierten Empfehlungen für alle denkbaren Konstellationen gegeben werden. Wir raten jedoch dazu, eine höhere initiale Dosis innerhalb dieses Bereiches (also in Richtung $25 \mathrm{mg} / \mathrm{Tag}$ Prednison-Äquivalent) bei Patienten mit einem hohen Rezidivrisiko und einem niedrigen Risiko für das Auftreten von Nebenwirkungen in Betracht zu ziehen. Dagegen sollten bei Patienten mit relevanten Komorbiditäten (z.B. Diabetes, Osteoporose, Glaukom usw.) und anderen Risikofaktoren für das Auftreten GCinduzierter Nebenwirkungen eine niedrigere Dosis innerhalb des Bereichs (also eher $15 \mathrm{mg} / \mathrm{Tag}$ ) bevorzugt werden. Der empfohlene Initialbereich in der Quellleitlinie ist mit $12,5-25 \mathrm{mg} / \mathrm{Tag}$ sogar noch weiter, aber im deutschen Sprachraum ist der Beginn mit 12,5 mg/Tag unüblich.

Empfehlung 2d (Dosisreduktion bzw. -anpassung). Die Glukokortikoid-Dosis soll kontinuierlich reduziert werden, basierend auf einem regelmäßigen Monitoring der Krankheitsaktivität des Patienten, der Laborparameter und des Auftretens von Nebenwirkungen.

Die folgenden Prinzipien zur Reduktion bzw. Anpassung der GlukokortikoidDosis werden empfohlen: Bei der initialen Reduktion sollte eine Dosis von $10 \mathrm{mg} / \mathrm{Tag}$ Prednison-Äquivalent innerhalb von 4 bis 8 Wochen erreicht werden. Danach sollte die tägliche Prednison-Dosis weiter um etwa $1 \mathrm{mg}$ alle 4 Wochen bis zum Absetzen reduziert werden.

Wird während des Absenkens der GCTherapie die Krankheit wieder aktiv (Rezidiv), sollte die Prednison-Dosis zumindest auf die Prä-Rezidiv-Dosis erhöht und dann schrittweise innerhalb von 4 bis 8 Wochen wieder reduziert werden auf die Dosis, bei der das Rezidiv auftrat. (Level ${ }^{*}$ )

Empfehlung 2e (Behandlungsdauer). Die Dauer der Glukokortikoid-Therapie soll für jeden PMR-Patienten individuell angepasst werden. Die Behandlungsdauer sollte so lang wie nötig, aber so kurz wie möglich sein. $\left(\right.$ Level $\left.^{*} 5\right)$

In Übereinstimmung mit den übergeordneten Prinzipien und den zur Erklärung für die Empfehlung 2c vorgetragenen Argumenten sollte sowohl die Absenkung der GC-Dosis im Behandlungsverlauf als auch die Dosierung im Falle eines Rezidivs sehr individualisiert bzw. patientenspezifisch und in Abhängigkeit von den Ergebnissen des regelmäßigen Monitorings erfolgen. Daher handelt es sich bei den Dosis- und Zeitangaben eher um Eckwerte, die Richtschnur des Handels sein können, wenngleich Therapieerfolge auch mit alternativen Behandlungsregimen erzielt werden können. So sei darauf hingewiesen, dass bei Patienten mit einer höheren initialen GC-Dosis von z. B. $25 \mathrm{mg} /$ Tag Prednison-Äquivalent in der Regel eine raschere Absenkung erfolgen kann als bei niedrigeren Initialdosierungen von z. B. nur $15 \mathrm{mg} / \mathrm{Tag}$.

Der oben genannte Begriff „Monitoring " umfasst sowohl klinische und laborchemische Verlaufskontrollen der PMR also auch die Beachtung der Empfehlungen zu Verlaufskontrollen unter Glukokortikoid-Therapie [21, 22]. Bezüglich der Knochengesundheit sind die aktuellen Empfehlungen des Dachverbandes Osteologie e. V. (DVO) zu beachten.

Empfehlung 3. Zusätzlich zur Glukokortikoid-Therapie sollte die Gabe von Methotrexat frühzeitig in Betracht gezogen werden, insbesondere bei Patienten mit einem hohen Risiko für Rezidive und/ oder für eine lange Therapiedauer sowie bei Patienten mit Risikofaktoren, Komorbiditäten und/oder Begleitmedikationen, bei denen Glukokortikoid-induzierte $\mathrm{Ne}$ benwirkungen mit höherer Wahrscheinlichkeit auftreten.

Methotrexat kann auch erwogen werden bei Patienten mit Rezidiv(en), unzureichendem Ansprechen auf Glukokortikoide oder bei Auftreten Glukokortikoidinduzierter Nebenwirkungen. (Level ${ }^{\star} 1$ )

Es gibt keine prototypische klinische Situation bei der PMR, die ohne jeden Zweifel die Anwendung von Methotrexat (MTX) erfordert. Vielmehr wird 
auch bei der Anwendung von MTX sehr individualisiert bzw. patientenspezifisch entschieden. In Betracht gezogen werden sollte MTX als Komedikation zu GC jedoch insbesondere bei Patientinnen [23-27], Patienten mit einer hohen BSG $(>40 \mathrm{~mm} / \mathrm{h})$ [28-33], peripherer Arthritis [34] und oder Komorbiditäten, die durch eine GC-Therapie ungünstig beeinflusst werden können. Es besteht allgemein Konsensus darüber, dass MTX als Komedikation auch bei Patienten (i) mit PMR Rezidiv(en), (ii) ohne ausreichende Reaktion auf eine GC-Therapie und (iii) mit GC-induzierten Nebenwirkungen infrage kommt. Grundlage für diese Aussagen sind Beobachtungen, die in sowohl randomisierten und kontrollierten Studien als auch in einer retrospektiven Studie gemacht worden sind. Die Ergebnisse dieser Studien waren teilweise widersprüchlich. Allerdings waren die Studien, die von einer guten Wirksamkeit von MTX berichteten (bezüglich Rezidivrate, kumulative GCDosis, Möglichkeit des Absetzens der GC), von deutlich höherer Qualität als die Studien, die zu negativen Ergebnissen kamen. Details dazu sind in der Quellleitlinie $[5,6]$ und in Übersichtsarbeiten $[1,3]$ zusammenfassend dargestellt und kommentiert. Bei Patienten mit eingeschränkter Nierenfunktion sollte MTX zurückhaltend und wenn, dann nur unter sorgfältig durchgeführten Verlaufskontrollen angewandt werden. Aktuell ist die Kosteneffektivität einer MTX-Anwendung bei Patienten mit PMR nicht klar, weitere Studien sind daher notwendig. Für die Anwendung von anderen konventionellen Basistherapeutika - wie sie z.B. in der Behandlung der rheumatoiden Arthritis eingesetzt werden können in Ermangelung von geeigneten Studiendaten keine Empfehlungen gemacht werden.

Empfehlung 4. PMR-Patienten sollen nicht mit TNF- $\alpha$-blockierenden Substanzen behandelt werden. $\mathrm{Zu}$ anderen Biologika inklusive Tocilizumab kann derzeit keine Empfehlung abgegeben werden. (Level $^{*}$ 1)

Von der Anwendung von TNF- $\alpha$ Hemmern wird abgeraten, weil es keinen Beweis für deren Wirksamkeit bei der PMR, wohl aber bekannte Daten zu deren potenziellen Nebenwirkungen und hohen Kosten gibt. Es liegt derzeit eine Studie zur Behandlung von PMRPatienten mit Tocilizumab vor, die ein positives Ergebnis zeigte [10]. Aufgrund der kleinen Fallzahl und der geringen methodischen Qualität kann daraus noch keine Empfehlung zum Einsatz von Tocilizumab bei der PMR abgeleitet werden kann. Für andere Biologika kann keine Empfehlung abgegeben werden, weil bisher keine prospektiven Studien publiziert worden sind. Allerdings laufen aktuell klinische Untersuchungen zur Wirkung von verschiedenen monoklonalen Antikörpern.

Empfehlung 5. Insbesondere älteren und/ oder gebrechlichen Patienten sollte zusätzlich zur medikamentösen Therapie ein individualisiertes Übungsprogramm angeboten werden. (Level ${ }^{\star} 5$ )

Es gibt keine Studien, die den positiven Wert von physiotherapeutischen Maßnahmen belegen. Dennoch stellen die Erhaltung von Muskelmasse und -funktion und die Reduktion des Sturzrisikos erstrebenswerte Ziele dar. Daher wird trotz der fehlenden Evidenz, aber auch dem dringenden Patientenwunsch folgend - ein individualisiertes Übungsprogramm empfohlen, was vornehmlich bei älteren und/oder gebrechlichen Patienten als nutzbringend angesehen wird.

Zusammenfassend bleibt zu konstatieren, dass zur Behandlung der PMR nach wie vor GC die Medikamente der ersten Wahl sind. Bei den meisten Patienten wird rasch eine Linderung der Beschwerden erzielt. Daher ist diese Therapie in der Praxis sehr gut etabliert, obwohl für Eckdaten, wie z. B. Initialdosis, Reduktionsschemata und Behandlungsdauer, die Evidenzlage schlecht ist. Das liegt u. a. auch daran, dass es sehr viele verschiedene Subgruppen von PMR-Patienten gibt. Trotzdem geben wir hier aktualisierte Empfehlungen, die auf den 2015 EULAR-ACR-Empfehlungen für die Behandlung der PMR beruhen, insofern einen internationalen Konsens darstellen. Durch die Arbeit der Leitliniengruppe wurden diese nicht nur aktualisiert, sondern auch auf die aktuelle Situation in Deutschland, Österreich und der Schweiz angepasst. Wir hoffen, dass es dadurch gelungen ist, eine Unterstützung und generelle Entscheidungshilfen bei der Behandlung von PMR-Patienten in der klinischen Praxis zur Verfügung zu stellen, auch wenn die individuelle Therapie immer patientenspezifisch erfolgen muss.

\section{Korrespondenzadresse}

Prof. Dr. F. Buttgereit

Medizinische Klinik mit Schwerpunkt

Rheumatologie und Klinische Immunologie,

Charité Universitätsmedizin Berlin

Charitéplatz 1, 10117 Berlin, Deutschland

frank.buttgereit@charite.de

Förderung. Die Erstellung dieser S3-Leitlinie wurde finanziell von der Deutschen Gesellschaft für Rheumatologie unterstützt. Die Arbeit der Leitlinienkommission erfolgte in Kooperation mit bzw. entsprechend den Vorgaben der Arbeitsgemeinschaft der Wissenschaftlichen Medizinischen Fachgesellschaften (AWMF).

\section{Einhaltung ethischer Richtlinien}

Interessenkonflikt. F. Buttgereit: Berater- und/oder Referentenhonorare durch die Firmen Horizon Pharma, Mundipharma Int Ltd. und Sanofi; Co-principal investigator/site investigator bei einer von Mundipharma durchgeführten, internationalen PMR-Studie zur Untersuchung der Wirkungen von MR-Prednison. T. Brabant: Berater- und/oder Referentenhonorare durch die Firmen Lilly, Novartis und Amgen. U. Kiltz: Berater-und/oder Referentenhonorare durch die Firmen AbbVie, Chugai, Grünenthal, Janssen, MSD, Novartis, Pfizer, Roche und UCB. Forschungsunterstützungen (unrestricted grant) durch AbbVie und Pfizer. D. Kyburz: Berater und/oder Referentenhonorare durch die Firmen AbbVie, BMS, Celgene, Lilly, Menarini, MSD, Novartis, Pfizer, Roche, UCB. M. Schneider: Honorare für Beratung, Vorträge und Drittmittel: AbbVie, AstraZeneca, BMS, Boehringer-Ingelheim, Chugai, GSK, Janssen-Cilag, Lilly, MSD, Novartis, Pfizer, Roche, Sanofi-Aventis, UCB. C. Dejaco: Berater-und/ oder Referentenhonorare durch die Firmen MSD, Pfizer, UCB, AbbVie, Roche, Novartis, Lilly, Celgene, Merck, Signatis Pharma und GSK. Forschungsunterstützung (unrestricted grant) durch Pfizer und MSD. H. Dinges, I. Hiemer, M. Kaplani, A. Reißhauer und C. Weseloh geben an, dass kein Interessenkonflikt besteht.

Dieser Beitrag beinhaltet keine von den Autoren durchgeführten Studien an Menschen oder Tieren.

\section{Literatur}

1. Dejaco C, Matteson EL, Buttgereit F (2016) Diagnostics and treatment of polymyalgia rheumatica. ZRheumatol 75(7):687-700

2. MuratoreF,Pazzola G, PipitoneN, SalvaraniC(2016) Recent advances in the diagnosis and treatment of polymyalgia rheumatica. Expert Rev Clin Immunol 12(10):1037-1045 
3. Buttgereit F, Dejaco C, Matteson EL, Dasgupta B (2016) Polymyalgia rheumatica and giant cell arteritis:a systematicreview.JAMA315(22):2442-2458

4. Matteson EL, Dejaco C (2017) Polymyalgia rheumatica. Ann Intern Med 166(9):ITC65-ITC80

5. Dejaco C, Singh YP, Perel P, Hutchings A, Camellino D, Mackie S et al (2015) 2015 recommendations for the management of polymyalgia rheumatica: a European League Against Rheumatism/American College of Rheumatology collaborative initiative. Ann Rheum Dis 74(10):1799-1807

6. Dejaco C, Singh YP, Perel P, Hutchings A, Camellino D, Mackie Set al (2015) 2015 recommendations for the management of polymyalgia rheumatica: a European League Against Rheumatism/American College of Rheumatology collaborative initiative. Arthritis Rheumatol 67(10):2569-2580

7. Guyatt GH, Oxman AD, Kunz R, Brozek J, AlonsoCoello P, Rind D et al (2011) GRADE guidelines 6. Rating the quality of evidence-imprecision.J Clin Epidemiol 64(12):1283-1293

8. Balshem $\mathrm{H}$, Helfand $M$, Schunemann $\mathrm{HJ}$, Oxman AD, Kunz R, Brozek J et al (2011) GRADE guidelines: 3. Rating the quality of evidence. J Clin Epidemiol 64(4):401-406

9. Dejaco C, Singh YP, Perel P, Hutchings A, Camellino D, Mackie $S$ et al (2015) Current evidence for therapeutic interventions and prognostic factors in polymyalgia rheumatica: a systematic literature review informing the 2015 European League Against Rheumatism/American College of Rheumatology recommendations for the management of polymyalgia rheumatica. Ann Rheum Dis 74(10):1808-1817

10. Lally L, Forbess L, Hatzis C, Brief Report SR (2016) A prospective open-label phase lla trial of tocilizumab in the treatment of polymyalgia rheumatica. Arthritis Rheumatol 68(10):2550-2554

11. Hancock AT, Mallen CD, Muller S, Belcher J, Roddy E, Helliwell T et al (2014) Risk of vascular events in patients with polymyalgia rheumatica. CMA 186(13):E495-E501

12. Yurdakul FG, Bodur $H$, Sivas $F$, Baskan $B$, Eser F, Yilmaz $O$ (2015) Clinical features, treatment and monitoring in patients with polymyalgia rheumatica. Arch Rheumatol 30(1):28-33

13. Bellan M, Boggio E, Sola D, Gibbin A, Gualerzi A, Favretto $S$ et al (2017) Association between rheumatic diseases and cancer: results from a clinical practice cohort study. Intern Emerg Med 12(5):621-627

14. Myklebust G, Wilsgaard T, Jacobsen BK, Gran JT (2002) No increased frequency of malignant neoplasms in polymyalgia rheumatica and temporal arteritis. A prospective longitudinal study of 398 cases and matched population controls.J Rheumatol 29(10):2143-2147

15. Haga HJ, Eide GE, Brun J, Johansen A, Langmark $F$ (1993) Cancer in association with polymyalgia rheumatica and temporal arteritis. J Rheumatol 20(8):1335-1339

16. Haugeberg G, Dovland H, Johnsen V (2002) Increased frequency of malignancy found in patients presenting with new-onset polymyalgic symptoms suggested to have polymyalgia rheumatica. Arthritis Rheum 47(3):346-347

17. Naschitz JE, Slobodin G, Yeshurun D, Rozenbaum M, Rosner I (1996) A polymyalgia rheumatica-like syndrome as presentation of metastatic cancer. JClin Rheumatol 2(6):305-308

18. NaschitzJE, Slobodin G, Yeshurun D, Rozenbaum M, Rosner I (1997) Atypical polymyalgia rheumatica as a presentation of metastatic cancer. Arch Intern Med 157(20):2381

19. Dasgupta B, Dolan AL, Panayi GS, Fernandes L (1998) An initially double-blind controlled 96 week trial of depot methylprednisolone against oral prednisolone in the treatment of polymyalgia rheumatica. BrJRheumatol 37(2):189-195

20. Cutolo M, Hopp M, Liebscher S, Dasgupta B, Buttgereit $F$ (2017) Modified-release prednisone for polymyalgia rheumatica: a multicentre, randomised, active-controlled, double-blind, parallel-group study. Rmd Open 3(1):e426

21. Miloslavsky EM, Naden RP, Bijlsma JW, Brogan PA, Brown ES, Brunetta P et al (2017) Development of a Glucocorticoid Toxicity Index (GTI) using multicriteria decision analysis. Ann Rheum Dis 76(3):543-546

22. van der Goes MC, Jacobs JW, Boers $M$, Andrews T, Blom-Bakkers MA, Buttgereit $F$ et al (2010) Monitoring adverse events of low-dose glucocorticoid therapy: EULAR recommendations for clinical trials and daily practice. Ann Rheum Dis 69(11):1913-1919

23. Dolan AL, Moniz C, Dasgupta B, Li F, Mackintosh C, Todd P et al (1997) Effects of inflammation and treatment on bone turnover and bone mass in polymyalgia rheumatica. Arthritis Rheum 40(11):2022-2029

24. Cimmino MA, Moggiana G, Montecucco C, Caporali R, Accardo S (1994) Long term treatment of polymyalgia rheumatica with deflazacort. Ann Rheum Dis 53(5):331-333

25. Cimmino MA, Parodi M, Caporali R, Montecucco C (2006) Is the course of steroid-treated polymyalgia rheumatica more severe in women? Ann N Y Acad Sci 1069:315-321

26. Ayoub WT, Franklin CM, Torretti D (1985) Polymyalgia rheumatica. Duration of therapy and long-term outcome. Am J Med 79(3):309-315

27. Barraclough K, Liddell WG, du Toit J, Foy C, Dasgupta B, Thomas M et al (2008) Polymyalgia rheumatica in primary care: a cohort study of the diagnostic criteria and outcome. Fam Pract 25(5):328-333

28. Kremers HM, Reinalda MS, Crowson CS, Zinsmeister AR, Hunder GG, Gabriel SE (2005) Relapse in a population based cohort of patients with polymyalgia rheumatica.J Rheumatol 32(1):65-73

29. Salvarani C, Boiardi L, Mantovani V, Ranzi A, Cantini F, Olivieri let al (1999) HLA-DRB1 alleles associated with polymyalgia rheumatica in northern Italy: correlation with disease severity. Ann Rheum Dis 58(5):303-308

30. Cantini F, Salvarani C, Olivieri I, Macchioni L, Ranzi A, Niccoli L et al (2000) Erythrocyte sedimentation rate and $\mathrm{C}$-reactive protein in the evaluation of disease activity and severity in polymyalgia rheumatica: a prospective follow-up study. Semin Arthritis Rheum 30(1):17-24

31. Lee JH, Choi ST, Kim JS, Yoon BY, Kwok SK, Kim HS et al (2013) Clinical characteristics and prognostic factors for relapse in patients with polymyalgia rheumatica (PMR). Rheumatol Int 33(6): $1475-1480$

32. Myklebust G, Gran JT (2001) Prednisolone maintenance dose in relation to starting dose in the treatment of polymyalgia rheumatica and temporal arteritis. A prospective two-year study in 273 patients. Scand J Rheumatol 30(5):260-267

33. Gonzalez-Gay MA, Rodriguez-Valverde V, Blanco $\mathrm{R}$, Fernandez-Sueiro JL, Armona J, Figueroa M et al (1997) Polymyalgia rheumatica without significantly increased erythrocyte sedimentation rate. A more benign syndrome. Arch Intern Med 157(3):317-320

34. Salvarani C, Cantini F, Macchioni P, Olivieri I, Niccoli L, Padula A et al (1998) Distal musculoskeletal manifestations in polymyalgia rheumatica: a prospective followup study. Arthritis Rheum 41(7):1221-1226

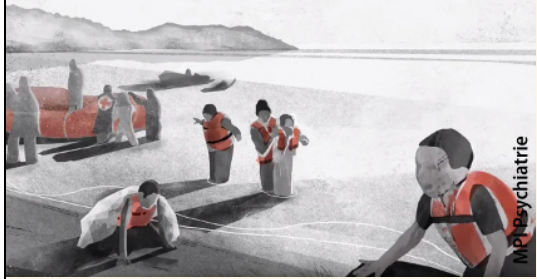

\section{Kurzfilm zu psychischen Symptomen nach Flucht}

Informationen für Helfer und Geflüchtete über mögliche Störungen nach Flucht und Migration

„Flucht und Migration" heißt der Kurzfilm des Max-Planck-Instituts für Psychiatrie (MPI), der mit Hilfe von Bildern zeigt, dass Schlafstörungen, Grübeln oder auch körperliche Beschwerden wie Kopfschmerzen oder Atemnot Symptome einer psychischen Erkrankung sein können. Der Film soll Betroffene entlasten und ermutigen, sich Hilfe zu suchen.

Psychische und körperliche Symptome müssen aber nicht unbedingt langfristig in eine psychiatrische Erkrankung münden. Sie können als nachvollziehbare Reaktion auf das Erlebte auch ohne Behandlung mit der Zeit abklingen. Auch das möchte der Film klar machen.

Der zwei Minuten lange Film liegt in sieben Sprachen vor: Deutsch, Englisch, Französisch, Italienisch, Arabisch, Dari und Kurdisch (Kurmandschi), in Vorbereitung sind Pashto, Tigrinya und Somali. Er kann hier angesehen und heruntergeladen werden: http://bit.ly/2FDTj14.

"Flucht und Migration" kann weitergeleitet werden, um möglichst viele Geflüchtete sowie ihre Helfer zu erreichen.

Der Film ist im Rahmen des Projektes RefPsych entstanden. Das MPI bietet Geflüchteten, Helfern und Interessierten darin Informationen zu psychischen Erkrankungen nach Flucht und Migration.

Quelle: Max-Planck-Institut für Psychiatrie (www.psych.mpg.de) 